05

\title{
Сдвиговые упругие свойства ВТСП керамики в области перехода в сверхпроводящую фазу
}

\author{
() А.И. Коробов, А.И. Кокшайский, Н.В. Ширгина, Н.И. Одина, А.А. Агафонов, В.В. Ржевский \\ Московский государственный университет им. М.В. Ломоносова, \\ 119991 Москва, Россия \\ e-mail: aikor42@mail.ru
}

Поступило в Редакцию 4 сентября 2019 г.

В окончательной редакции 6 декабря 2019 г.

Принято к публикации 19 декабря 2019 г.

Приведены результаты исследований сдвиговых упругих свойств высокотемпературной сверхпроводящей (ВТСП) керамики $\mathrm{YBa}_{2} \mathrm{Cu}_{3} \mathrm{O}_{7-x}$ в окрестностях фазового перехода в сверхпроводящее состояние при температуре $91.3 \mathrm{~K}$, а также при комнатной температуре $293 \mathrm{~K}$. В области температуры фазового перехода впервые обнаружено локальное увеличение сдвигового нелинейного акустического параметра $N$. Проведенные экспериментальные исследования линейных и нелинейных упругих свойств ВТСП керамики $\mathrm{YBa}_{2} \mathrm{Cu}_{3} \mathrm{O}_{7-x}$ в области сверхпроводящего перехода показали, что электронный фазовый переход оказывает существенное влияние на ее упругие свойства. На основе результатов экспериментальных измерений были определены линейные и нелинейные упругие параметры как при комнатной температуре, так и в области температуры фазового перехода.

Ключевые слова: скорость ультразвуковых волн, сверхпроводящая керамика, структурная упругая нелинейность, нелинейный сдвиговый упругий параметр.

DOI: $10.21883 /$ JTF.2020.06.49282.309-19

\section{Введение}

Со времени открытия высокотемпературных сверхпроводников (ВТСП) одним из наиболее интенсивно исследуемых материалов является ВТСП соединение $\mathrm{YBa}_{2} \mathrm{Cu}_{3} \mathrm{O}_{7-x}[1,2]$. Интерес к этому материалу вызван сравнительно высокой температурой фазового перехода $T_{c}$, равной приблизительно $91 \mathrm{~K}$ (превышающей температуру жидкого азота $T=77.4 \mathrm{~K}$ ) в сверхпроводящее состояние, относительно простой технологией его изготовления, возможностью его применения в различных областях электроники и электроэнергетики, например, при создании магнитных бесконтактных подшипников для накопителей энергии, при изготовлении объемных СВЧ резонаторов, а также при изготовлении электромоторов. Сверхпроводящие магнитные подшипники для быстро вращающихся конструкций, таких как турбины, маховики, двигатели и генераторы, являются одним из наиболее перспективных применений для ВТСП соединения $\mathrm{YBa}_{2} \mathrm{Cu}_{3} \mathrm{O}_{7-x}$ [3]. Керамика $\mathrm{YBa}_{2} \mathrm{Cu}_{3} \mathrm{O}_{7-x}$ представляет собой микрокристаллическую структуру, поэтому при эксплуатации изделий из нее могут возникать различные дефекты, в том числе микротрещины, влияющие на механическую прочность устройств, изготовленных из этой керамики. Вместе с тем существование в керамике $\mathrm{YBa}_{2} \mathrm{Cu}_{3} \mathrm{O}_{7-x}$ ряда фаз и фазовых переходов, зависящих от температуры, от содержания кислорода, неупорядоченности кислородной подрешетки $[2,4-8]$, делает необходимым для изучения ее упругих свойств применение структурно-чувствительных методов. К таким методам относятся измерения коэффициента теплово- го расширения $[9,10]$, ультразвуковые измерения [11], с помощью которых можно проводить эффективную диагностику ВТСП керамики $\mathrm{YBa}_{2} \mathrm{Cu}_{3} \mathrm{O}_{7-x}$. Измерения коэффициента теплового расширения керамики в $[9,10]$ проводились рентгеновским методом. Очевидно, что проводить периодическую диагностику промышленных изделий конечных размеров из керамики $\mathrm{YBa}_{2} \mathrm{Cu}_{3} \mathrm{O}_{7-x}$ в сверхпроводящем состоянии путем измерения коэффициента теплового расширения представляется довольно трудной задачей. Более перспективными методами неразрушающей диагностики конструкций конечных размеров из ВТСП керамики в сверхпроводящем состоянии являются ультразвуковые методы [12]. Анализ результатов исследований упругих свойств ВТСП керамик [2] показывает, что линейные сдвиговые свойства ВТСП керамик исследованы недостаточно, а исследования нелинейных упругих свойств практически отсутствуют. Особый интерес представляют нелинейные акустические эффекты, возникающие в ВТСП керамике $\mathrm{YBa}_{2} \mathrm{Cu}_{3} \mathrm{O}_{7-x}$ при распространении в ней гармонической сдвиговой акустической волны конечной амплитуды [11]. Проведенные авторами настоящей работы экспериментальные измерения скорости продольных и сдвиговых объемных акустических волн (ОАВ) в исследованных образцах ВТСП керамики $\mathrm{YBa}_{2} \mathrm{Cu}_{3} \mathrm{O}_{7-x}$ не обнаружили зависимости величины скорости ОАВ от направления распространения в образце. Это указывает на то, что микрокристаллическая керамика $\mathrm{YBa}_{2} \mathrm{Cu}_{3} \mathrm{O}_{7-x}$ является упругоизотропным материалом. Как показано в [7], вследствие высокой симметрии в изотропных твердых телах без дефектов генерация сдвиговой акустической 
гармоники запрещена. Однако в изотропных твердых телах кроме классической нелинейности, связанной с ангармонизмом кристаллической решетки, может наблюдаться неклассическая, структурная нелинейность, обусловленная наличием в микрокристаллическом изотропном твердом теле различных дефектов, в том числе микротрещин, при этом структурная нелинейность значительно превышает классическую. Экспериментально установлено, что структурная нелинейность зависит от количества структурных дефектов: с увеличением количества дефектов структурная нелинейность увеличивается. Первая ключевая работа по изучению влияния дефектной структуры на нелинейные упругие свойства поли- и монокристаллов металлов была выполнена в 1963 году на кафедре акустики физического факультета МГУ $[13,14]$. В этой работе экспериментально наблюдалась генерация второй сдвиговой гармоники в изотропных поликристаллических металлах: алюминии, магниево-алюминиевом сплаве МА-8. В результате проведенных экспериментов было установлено, что наличие неоднородностей и дефектов (дислокаций, микротрещин, границ микрозерен, локальных внутренних напряжений) существенно изменяет сдвиговые нелинейные упругие свойства твердых тел: в них появляется сдвиговая упругая структурная нелинейность и наблюдается генерация второй сдвиговой гармоники. При этом было отмечено, что с увеличением дефектности материала увеличивается сдвиговая упругая нелинейность в диагностируемом материале. Наличие сдвиговой гармоники упругой нелинейности в материале является диагностическим признаком и свидетельствует о наличии в нем дефектной структуры $[11,14]$, поэтому периодическое измерение второй сдвиговой гармоники в деталях промышленных устройств, изготовленных из керамики $\mathrm{YBa}_{2} \mathrm{Cu}_{3} \mathrm{O}_{7-x}$, находящейся в сверхпроводящем состоянии, позволит получить информацию о накоплении дефектов в этих устройствах.

Целью настоящей работы является экспериментальное исследование линейных и нелинейных сдвиговых упругих свойств ВТСП керамики $\mathrm{YBa}_{2} \mathrm{Cu}_{3} \mathrm{O}_{7-x}$ в сверхпроводящем состоянии, а также при температуре $293 \mathrm{~K}$.

\section{Экспериментальные образцы, установка и методика измерений}

Для проведения экспериментальных исследований использовались образцы ВТСП керамики $\mathrm{YBa}_{2} \mathrm{Cu}_{3} \mathrm{O}_{7-x}$, изготовленные в Национальном исследовательском центре „Курчатовский институт“. Эта керамика используется в промышленных приложениях, например, при изготовлении электромоторов. Из исходной заготовки с пористостью $10 \%$ были изготовлены три образца в форме прямоугольных параллелепипедов с размерами $36.22 \times 9.79 \times 7.19 \mathrm{~mm}$. Противоположные грани образцов были плоскопараллельны и тщательно полировались. Плотность образцов была равна $5690 \pm 70 \mathrm{~kg} / \mathrm{m}^{3}$.
На рис. 1, $a$ приведена микрофотография образца, полученная с помощью электронного микроскопа с коэффициентом увеличения $24971 \times$. На рис. $1, a$ видно, что образец состоит из микрокристаллов различной формы с характерными размерами порядка $1-2 \mu \mathrm{m}$. Также в образце обнаружена пора.

Ультразвуковые измерения в интервале температур от 78 до $300 \mathrm{~K}$ проводились с использованием автоматизированной экспериментальной ультразвуковой установки. Установка состоит из персонального компьютера (ПК), ультразвуковой системы Ritec RAM-5000, работающей в импульсном режиме, электрической схемы для измерения сопротивления образца методом четырехточки (на рис. 1 не показана), низкотемпературной термостатируемой акустической ячейки с исследуемым образцом, дьюара с жидким азотом и системы для измерения температуры образца с помощью термопары. Постоянное напряжение, пропорциональное температуре образца, с термопары поступало на контроллер Stanford Research System SR630, и после усиления и оцифровки 12 разрядным аналогоцифровым преобразователем сигнал подавался в ПК.

Акустическая ячейка с экспериментальным образцом опускалась в дьюар с жидким азотом. Ячейка состояла из кристаллодержателя, в котором был закреплен исследуемый образец с термопарой. Кристаллодержатель с образцом помещался в полый герметичный толстостенный цилиндр из бронзы. Бронза обладает высокими коэффициентами тепловодности и теплоемкости, что позволяло предотвратить неоднородность температуры в исследуемом образце. Температура образца изменялась путем плавного перемещения ячейки в парах азота. Скорость изменения температуры во всем диапазоне температур не превышала $0.5 \mathrm{~K} / \mathrm{min}$, а в окрестности сверхпроводящего перехода менее $0.1 \mathrm{~K} / \mathrm{min}$.

Для одновременного исследования линейных и нелинейных упругих свойств твердых тел в экспериментальной установке были реализованы следующие экспериментальные методики:

а) импульсный метод измерения скорости и поглощения ультразвуковых волн [15];

б) спектральный метод для определения нелинейных упругих свойств твердых тел, основанный на исследовании эффективности генерации высших акустических гармоник при распространении акустических волн конечной амплитуды в исследуемом образце [8].

Для возбуждения сдвиговых волн в образце керамики использовалась пластинка из пьезоэлектрического кристалла ниобата лития $\mathrm{YZ}-17^{\circ}$ среза с резонансной чистотой $5 \mathrm{MHz}$. Для приема сигнала второй упругой сдвиговой гармоники на частоте $2 f=10 \mathrm{MHz}$ использовалась аналогичная пластинка с резонансной частотой $10 \mathrm{MHz}$. Эта пластинка также использовалась и для приема ультразвукового сигнала на частоте $5 \mathrm{MHz}$. Пластинки крепились к противоположным полированным плоскопараллельным сторонам образца. Акустический 

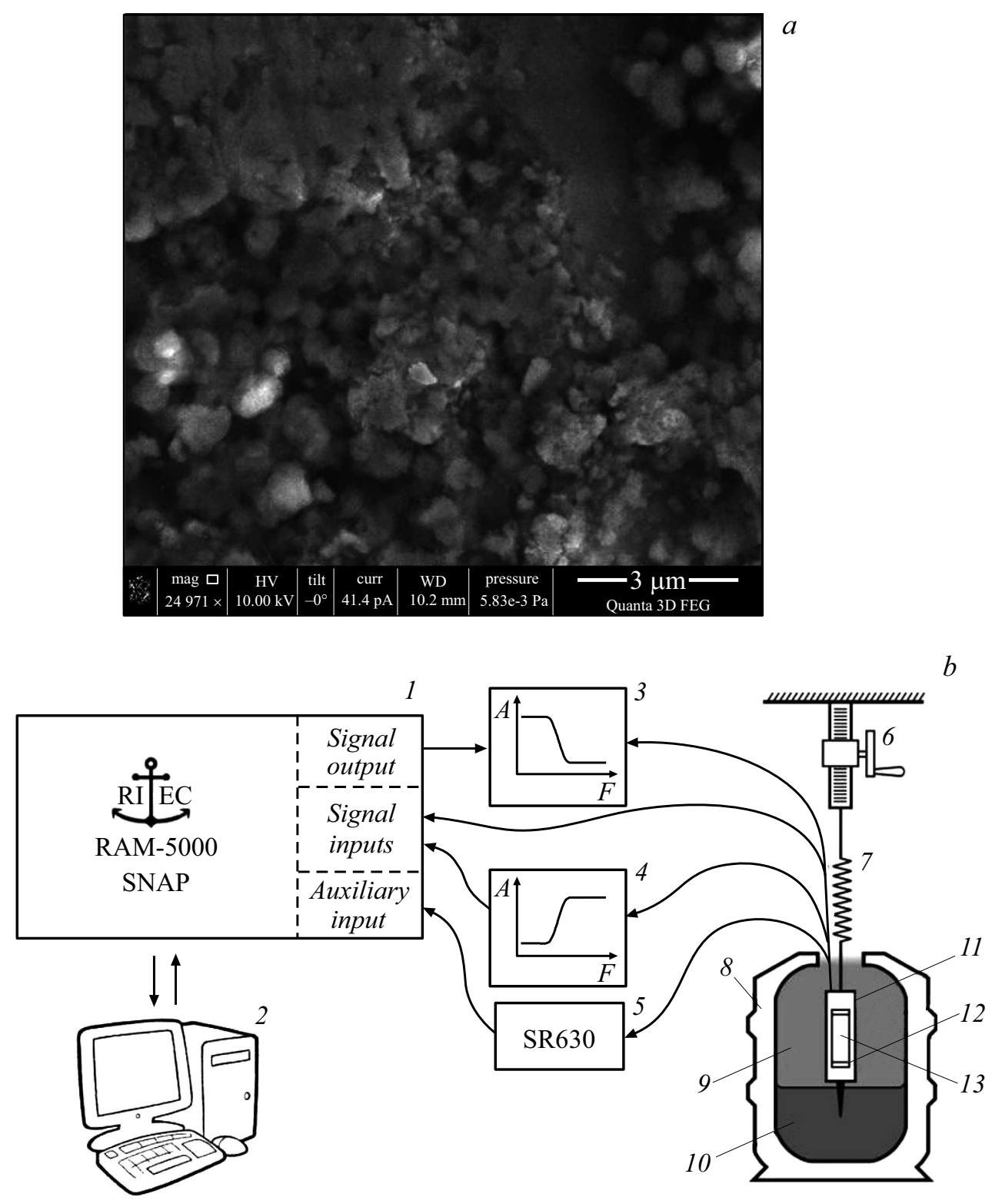

Рис. 1. $a-$ фотография образца, сделанная на электронном микроскопе; $b-$ экспериментальная установка для проведения низкотемпературных акустических измерений: 1 - ультразвуковая система Ritec RAM-5000, 2 - ПК, 3 - фильтр высоких частот, 4 - фильтр низких частот, 5 - многоканальный измеритель температуры Stanford Research System SR630, 6 - ручная лебедка с миллиметровой подачей, 7 - упругая виброразвязка, 8 - сосуд Дьюара, 9 - пары азота, 10 - жидкий азот, $11-$ массивная латунная емкость, 12 - пьезоэлектрические преобразователи, 13 - образец.

контакт между пьезоэлектрическими преобразователями и образцом в интервале температур (78-300) K осуществлялся с помощью силиконового масла, обладающего сдвиговой упругостью и незамерзающего при температуре жидкого азота. С ультразвукового измерительного комплекса на излучающий пьезоэлектрический преобразователь подавался высокочастотный импульс с частотой заполнения $f=5 \mathrm{MHz}$ и амплитудой до $500 \mathrm{~V}$, длительностью (3-5) $\mu \mathrm{s}$, который возбуждал в образце упругую волну. Прошедшая через образец волна регистрировалась приемным преобразователем. Спектр прошедшего через образец акустического сигнала содер- жал, кроме сигнала зондирующей волны $A_{f}$ на частоте $f=5 \mathrm{MHz}$, сигнал второй гармоники $A_{2 f}$ на частоте $2 f=10 \mathrm{MHz}$. Для выделения сигналов первой и второй гармоник из спектра акустической волны, прошедшей через образец, применялись фильтры нижних и высоких частот. Отфильтрованные сигналы основной частоты и второй гармоники подавались на отдельные приемные входы ультразвукового комплекса. Это позволяло по двум независимым каналам проводить одновременное измерение амплитуды $A$ и фазы $\varphi$ сигналов первой и второй гармоник в зависимости от амплитуды зондирующего сигнала при различных температурах исследуе- 
мого образца. Регистрация экспериментальных данных и их последующая математическая обработка осуществлялась в ПК.

\section{Экспериментальные результаты и их обсуждение}

В приготовленных образцах керамики $\mathrm{YBa}_{2} \mathrm{Cu}_{3} \mathrm{O}_{7-x}$ импульсным ультразвуковым методом на прохождение [16] вдоль трех ортогональных направлений, параллельных трем ребрам параллелепипеда, были измерены скорости продольных и сдвиговых объемных акустических волн $(\mathrm{OAB})$ при комнатной температуре $T=300 \mathrm{~K}$. Численные значения как для продольных, так и для сдвиговых скоростей ОАВ в образце не зависели от направления распространения ОАВ. Проведенные измерения скорости ОАВ дали возможность считать исследуемые образцы упругоизотропным твердым телом. Также была измерена плотность образцов ВТСП $\rho_{0}=5690 \pm 70 \mathrm{~kg} / \mathrm{m}^{3}$. На основании этих измерений были рассчитаны коэффициенты упругости второго порядка в исследованной ВТСП керамике (см. таблицу).

В изотропных твердых телах имеется 12 отличных от нуля коэффициентов упругости второго порядка (КУВП), но только 2 из них являются независимыми. Обычно такими принято считать коэффициенты $C_{11}$ и $C_{44}$. В таблице приведены результаты измерения скоростей продольной и сдвиговой $\mathrm{OAB}$, значение плотности керамики $\mathrm{YBa}_{2} \mathrm{Cu}_{3} \mathrm{O}_{7-x}$ и рассчитанные по формулам (1) и (2) значения КУВП в исследованном образце:

$$
\begin{aligned}
& C_{11}=\rho_{0} V_{L}^{2}, \\
& C_{44}=\rho_{0} V_{T}^{2},
\end{aligned}
$$

где $\rho_{0}$ - плотность образца, $V_{L}$ - продольная скорость, $V_{T}$ - сдвиговая скорость ОАВ.

Для описания упругих свойств изотропных твердых тел наряду с тензорными коэффициентами $C_{11}$ и $C_{44}$ часто используются скалярные величины: $\lambda, \mu-$ коэффициенты Ламе, $S i j$ - модуль податливости второго порядка, $E-$ модуль Юнга, $K-$ коэффициент всестороннего сжатия, $\sigma$ - коэффициент Пуассона. Любая пара этих коэффициентов однозначно описывает упругие свойства твердых тел. Экспериментально определенные коэффициенты $C_{11}$ и $C_{44}$ позволили рассчитать коэффициенты Ламэ $\lambda, \mu$, модуль Юнга $E$, коэффициент Пуассона $\sigma$, коэффициент всестороннего сжатия $K$ в ВТСП керамике.

Значение этих коэффициентов в ВТСП керамике приведено в таблице. Методика расчета этих коэффициентов приведена в [17].

Для определения температуры сверхпроводящего перехода из ВТСП керамики $\mathrm{YBa}_{2} \mathrm{Cu}_{3} \mathrm{O}_{7-x}$ был приготовлен образец размером $1 \times 1 \times 7 \mathrm{~mm}$. Сопротивление образца $R$ в интервале температур $(78-300) \mathrm{K}$ было

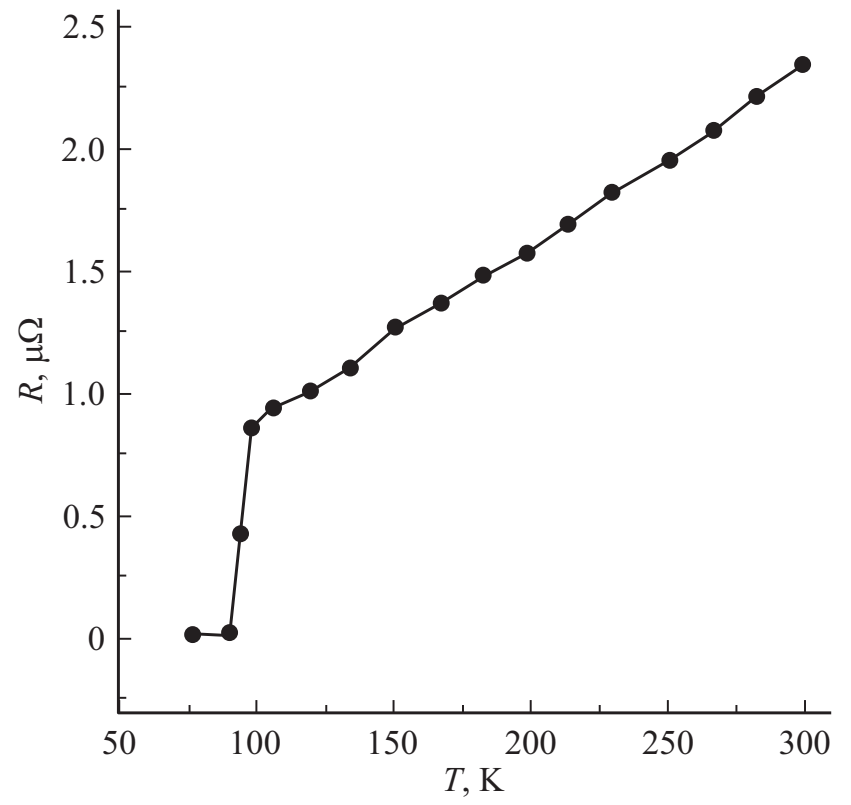

Рис. 2. Температурная зависимость сопротивления образца керамики $\mathrm{YBa}_{2} \mathrm{Cu}_{3} \mathrm{O}_{7-x}$.

измерено методом четырехточки при токе через образец $J=1 \mathrm{~mA}$. Эти измерения зарегистрировали переход керамики $\mathrm{YBa}_{2} \mathrm{Cu}_{3} \mathrm{O}_{7-x}$ в сверхпроводящее состояние при температуре $T_{c}=(91.3 \pm 0.1) \mathrm{K}$ (ширина перехода $\sim 1 \mathrm{~K})$ (рис. 2).

Линейные упругие свойства керамики в интервале температур $(79-300) \mathrm{K}$ исследовались импульсным методом путем измерения скорости продольных и сдвиговых ОАВ. С понижением температуры на фоне монотонного роста у скоростей как продольных, так и сдвиговых волн обнаружено аномальное поведение при переходе материала в сверхпроводящее состояние при температуре $T_{c}=91.3 \mathrm{~K}$. Особенно тщательно нами были измерены температурные зависимости скоростей ОАВ в области сверхпроводящего перехода (рис. 3).

В случае продольных волн при $T=T_{c}=91.3 \mathrm{~K}$ отмечены аномалии как самой зависимости, так и ее производной по температуре (рис. 3). Для оценки величин наблюдаемых скачков скорости кривая зависимости была аппроксимирована отрезками прямых с использованием метода наименьших квадратов на участках выше $(T=92.5-94 \mathrm{~K})$ и ниже $(T=88-90 \mathrm{~K})$ температуры перехода, после чего обе прямые были экстраполированы до точки $T_{c}$. Согласно оценкам, скачок относительного изменения скорости продольной волны $V_{1} \frac{\Delta V_{l}}{V_{l}}\left(T_{c}\right)=-1.5 \cdot 10^{-4}$ (рис. $3, a$ ), при этом изменение производной было равно $\Delta\left[\frac{d}{d T}\left(\frac{\Delta V_{l}}{V_{l}}\right)\right]=1.3 \cdot 10^{-4} / K$.

Для зависимости скорости сдвиговой волны от температуры $\frac{\Delta V_{t}}{V_{t}}\left(T_{c}\right)$, аппроксимированной аналогично $\frac{\Delta V_{l}}{V_{l}}(T)$, в пределах ошибки измерений скачка величины $\frac{\Delta V_{t}}{V_{t}}$ не наблюдалось, величина же скач- 
Упругие параметры ВТСП керамики $\mathrm{YBa}_{2} \mathrm{Cu}_{3} \mathrm{O}_{7-x}$ при температуре $T=300 \mathrm{~K}$

\begin{tabular}{c|c|c|c|c|c|c|c|c|c|c}
\hline $\begin{array}{c}\text { Плотность }, \\
\mathrm{kg} / \mathrm{m}^{3}\end{array}$ & $V_{L}, \mathrm{~m} / \mathrm{s}$ & $V_{S}, \mathrm{~m} / \mathrm{s}$ & $C_{11}, \mathrm{GPa}$ & $C_{44}=\mu \mathrm{GPa}$ & $S_{11}$ & $S_{12}$ & $K, \mathrm{GPa}$ & $E, \mathrm{GPa}$ & $\sigma, \mathrm{Pa}$ & $\lambda, \mathrm{GPa}$ \\
\hline $5690 \pm 70$ & $4530 \pm 70$ & $2630 \pm 40$ & $116.74 \pm 3.48$ & $39.35 \pm 1.18$ & $\begin{array}{c}(1.02 \\
\pm 0.03) \cdot 10^{-11}\end{array}$ & $\begin{array}{c}(-2.5 \\
\pm 0.07) \cdot 10^{-12}\end{array}$ & $64.2 \pm 1.9$ & $98 \pm 3$ & $0.25 \pm 0.07$ & $38 \pm 1$
\end{tabular}
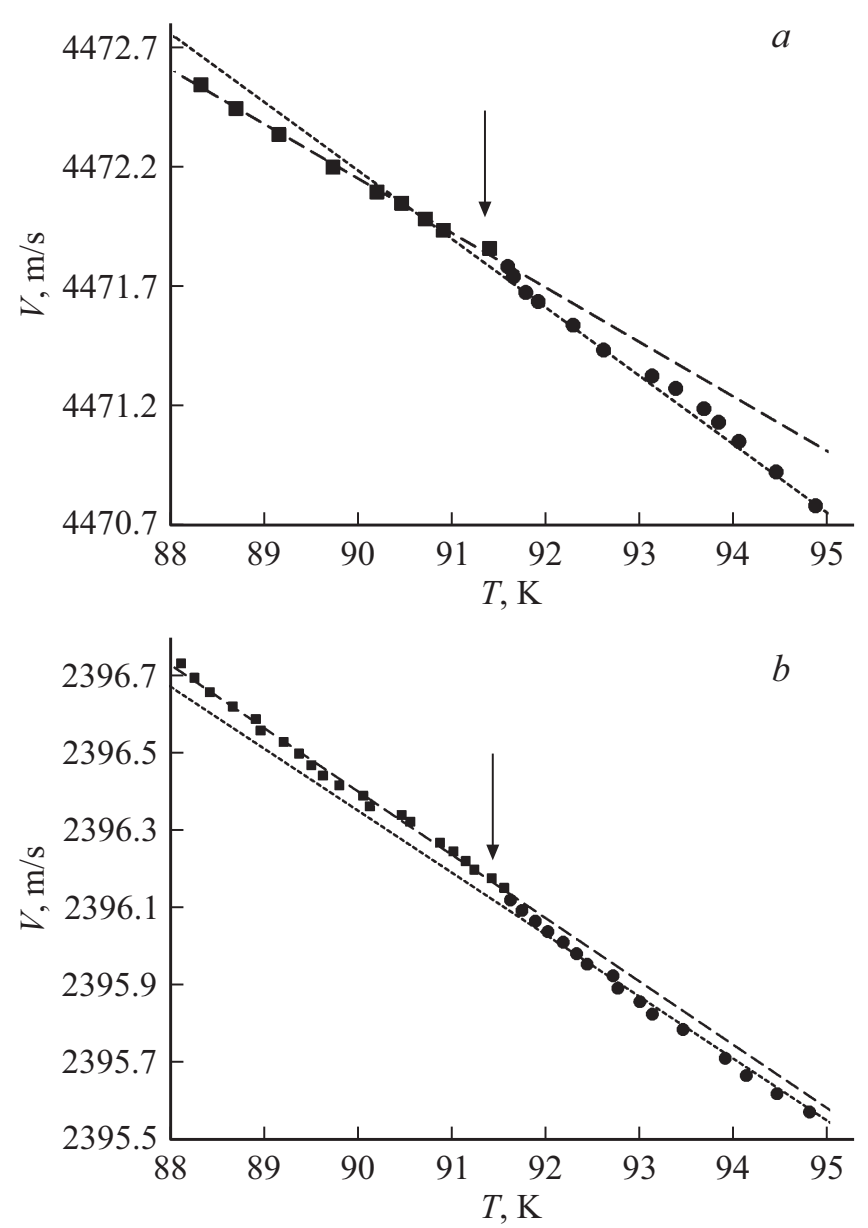

Рис. 3. Температурные зависимости продольных $(a)$ и сдвиговых $(b)$ OАВ в ВТСП керамике $\mathrm{YBa}_{2} \mathrm{Cu}_{3} \mathrm{O}_{7-x}$ в области температуры фазового сверхпроводящего перехода при $T_{c}=91.3 \mathrm{~K}$.

ка производной при $T=T_{c}$ составила (рис. $3, b$ ) $\Delta\left[\frac{d}{d T}\left(\frac{\Delta V_{l}}{V_{l}}\right)\right]=1.02 \cdot 10^{-4} 1 / K$.

Одновременно импульсным методом было исследовано поглощение сдвиговой акустической волны в образце в зависимости от температуры в ВТСП керамике $\mathrm{YBa}_{2} \mathrm{Cu}_{3} \mathrm{O}_{7-x}$ в области фазового перехода. Поглощение упругих волн вычислялось по формуле

$$
\alpha=\frac{1}{l} \ln \left(\frac{A_{i}}{A_{i+1}}\right),
$$

где $l$ - длина образца, $A_{i}$ - амплитуда первого прошедшего импульса, $A_{i+1}$ - амплитуда второго импульса в серии упругих импульсов, прошедших через образец. Измеренная зависимость коэффициента поглощения сдвиговой упругой волны от температуры в окрестности фазового перехода при $T_{c}=91.3 \mathrm{~K}$ приведена на рис. 4. Как видно из приведенной на рис. 4 зависимости, с увеличением температуры наблюдается уменьшение поглощения упругой сдвиговой волны, однако вблизи точки фазового перехода $91.3 \mathrm{~K}$ наблюдается локальное увеличение коэффициента поглощения с 0.014 до $0.0151 / \mathrm{m}$.

Нелинейные сдвиговые упругие свойства ВТСП керамики $\mathrm{YBa}_{2} \mathrm{Cu}_{3} \mathrm{O}_{7-x}$ в области температуры сверхпроводящего фазового перехода при $T_{c}=91.3 \mathrm{~K}$ исследовались спектральным методом путем измерения эффективности генерации второй сдвиговой упругой гармоники в изучаемой керамике.

Этот метод заключается в следующем. В спектре гармонической сдвиговой ОАВ конечной амплитуды $A$, прошедшей через исследуемый твердотельный образец, кроме волны основной частоты $A_{f}=A_{1} \sin (\omega t-k l)$, наблюдалась вторая гармоника ОАВ $A_{2 f}=A_{2} \sin [2(\omega t-k l)]$ :

$$
A=A_{1} \sin (\omega t-k l)+A_{2} \sin [2(\omega t-k l)] .
$$

Генерация второй сдвиговой гармоники в изотропном образце связана с неклассической структурной нелинейностью, обусловленной наличием в исследуемом образце мелкокристаллической структуры (рис. 1,a). Зависимость амплитуды второй гармоники $A_{2}$ от амплитуды ОАВ первой гармоники $A_{1}$ в (4) определяется

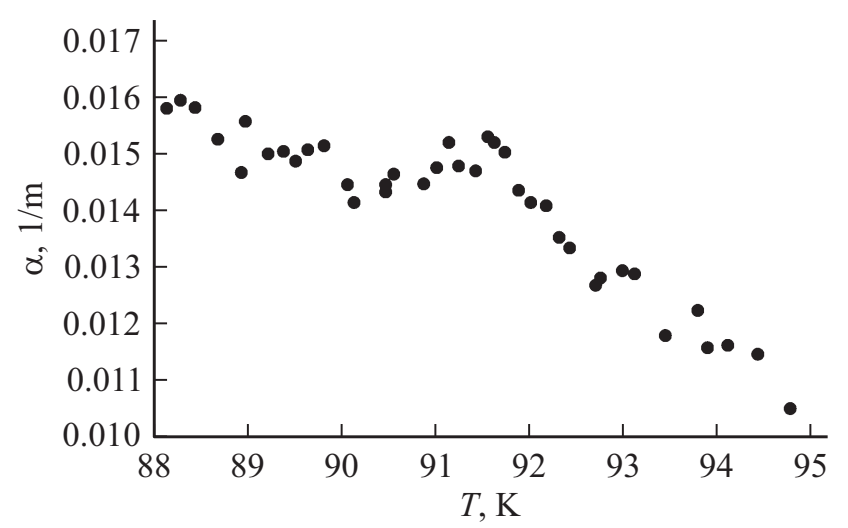

Рис. 4. Температурная зависимость коэффициента поглощения $\alpha$ для сдвиговых упругих волн от температуры в ВТСП керамике $\mathrm{YBa}_{2} \mathrm{Cu}_{3} \mathrm{O}_{7-x}$ в области температуры сверхпроводящего фазового перехода при $T_{c}=91.3 \mathrm{~K}$. 

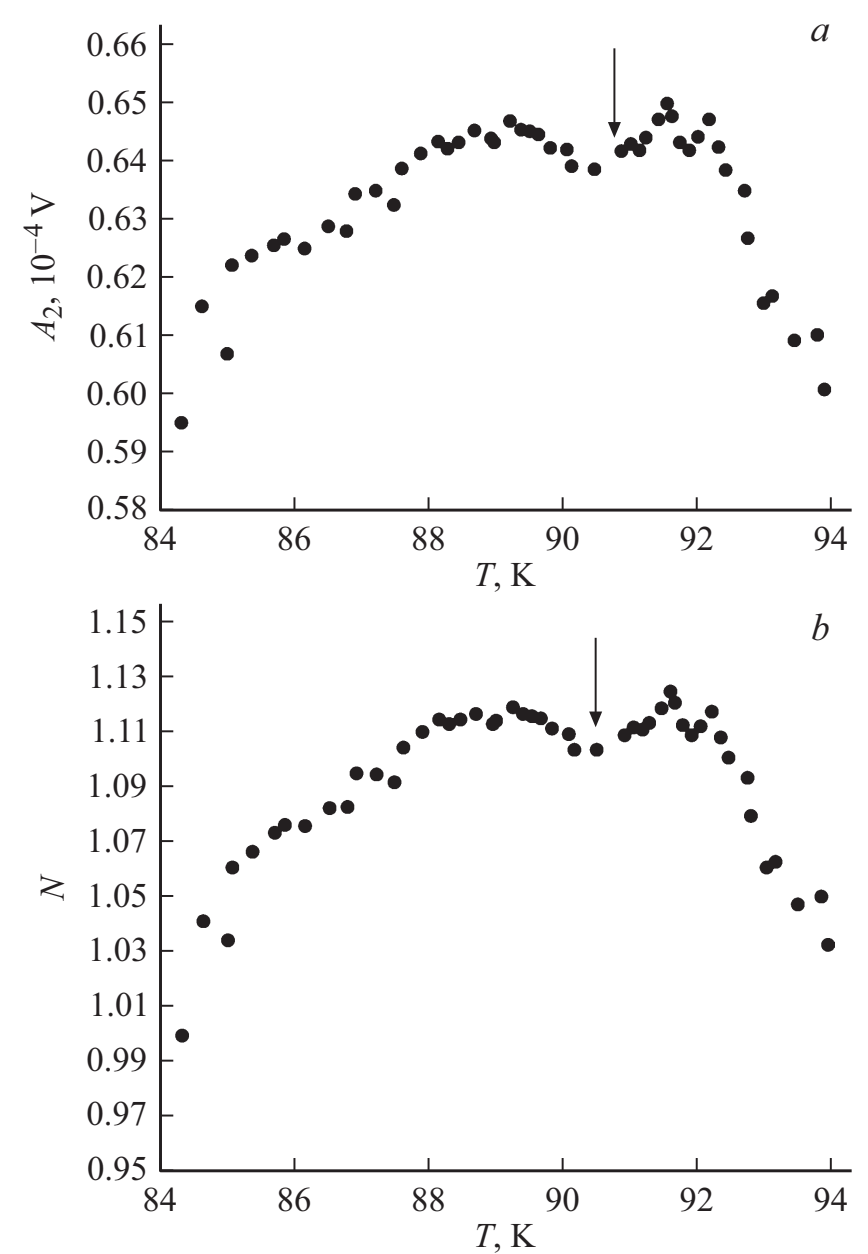

Рис. 5. Температурные зависимости: $a-$ амплитуды второй сдвиговой гармоники $A_{2} ; b-$ нормированного нелинейного упругого параметра второго порядка $N(T)$ для сдвиговых упругих волн в ВТСП керамике вблизи температуры сверхпроводящего перехода при $T_{c}=91.3 \mathrm{~K}$.

уравнением [13]

$$
A_{2}=\left[\left(N k^{2} L\right) A_{1}^{2}\right] / 8,
$$

где $k=\omega / V_{T}-$ волновой вектор, $V_{T}-$ скорость сдвиговой акустической волны, $\omega-$ частота первой упругой гармоники, $L-$ длина области взаимодействия, $N-$ нелинейный акустический параметр для ОАВ в твердом теле. Экспериментальные исследования температурных зависимостей амплитуды второй гармоники $A_{2}(T)$ от амплитуды первой гармоники $A_{1}(T)$ ОАВ позволяют определить температурную зависимость сдвигового нелинейного акустического параметра $N(T)$, численно характеризующего упругую нелинейность образца.

Из уравнения (5) следует

$$
N(T)=\left[8 A_{2}(T)\right] /\left[\left(k_{f}^{2} L\right) A_{1}^{2}(T)\right] .
$$

На рис. 5 приведены экспериментальные результаты температурных зависимостей амплитуды второй сдвиговой упругой гармоники и акустического нелинейного параметра $N(T)$ для сдвиговых упругих волн второго порядка в области сверхпроводящего перехода при $T_{c}=91.3 \mathrm{~K}$, нормированного на его значения при температуре $84 \mathrm{~K}$.

Как следует из рис. 5, в окрестности температуры $T_{c}=91.3 \mathrm{~K}$, соответствующей сверхпроводящему фазовому переходу, наблюдалось локальное увеличение сдвигового нелинейного акустического параметра: его величина увеличилась примерно в 1.13 раза.

Увеличение поглощения основной частоты, амплитуды второй сдвиговой упругой гармоник и нелинейного акустического параметра (рис. 4, 5) вблизи температуры фазового перехода $T_{c}=91.3 \mathrm{~K}$ связаны со структурными изменениями в системе, сопровождающими сверхпроводящий переход. Особенности в поведении коэффициента теплового расширения при температуре фазового перехода были обнаружены в $[9,10]$, которые авторы связывают с изменением структуры образца.

\section{Заключение}

В работе исследованы образцы ВТСП керамики $\mathrm{YBa}_{2} \mathrm{Cu}_{3} \mathrm{O}_{7-x}$, изготовленной в Национальном исследовательском центре „Курчатовский институт“. Эта керамика используется при изготовлении электромоторов. В ВТСП керамике $\mathrm{YBa}_{2} \mathrm{Cu}_{3} \mathrm{O}_{7-x}$ при температуpe $293 \mathrm{~K}$ были измерены скорости продольных и сдвиговых волн параллельно ребрам образца. На основании результатов измерений величин скоростей продольных и сдвиговых волн был сделан вывод, что исследуемые образцы являются упруго изотропными. Проведенные измерения скорости ОАВ и плотности образцов позволили определить все независимые компоненты тензоров упругости второго порядка. В интервале температур (78-300) K методом четырехточки измерена температурная зависимость сопротивления образца ВТСП керамики $\mathrm{YBa}_{2} \mathrm{Cu}_{3} \mathrm{O}_{7-x}$. При температуре $T=91.3 \mathrm{~K}$ в исследуемом образце был обнаружен фазовый переход в сверхпроводящее состояние. Несмотря на то, что образец являлся упруго изотропным, в нем наблюдалась генерация второй сдвиговой гармоники, которая связана с микрокристаллической структурой образца. В окрестности температуры фазового перехода впервые обнаружено увеличение сдвигового нелинейного акустического параметра $N$, характеризующего нелинейные сдвиговые упругие свойства ВТСП керамики $\mathrm{YBa}_{2} \mathrm{Cu}_{3} \mathrm{O}_{7-x}$, которое объясняется изменением внутренней структуры образца. Изменение структуры образца ВТСП керамики $\mathrm{YBa}_{2} \mathrm{Cu}_{3} \mathrm{O}_{7-x}$ в области фазового перехода было ранее зарегистрировано при исследовании рентгеновским методом коэффициента теплового расширения в $[9,10]$.

Генерация второй сдвиговой гармоники может служить диагностическим признаком изменения внутренней структуры образца, в том числе появления микротрещин. 
Проведенные исследования нелинейных упругих сдвиговых свойств ВТСП керамики $\mathrm{YBa}_{2} \mathrm{Cu}_{3} \mathrm{O}_{7-x}$ показывают, что они могут быть использованы при неразрушающей акустической диагностике образцов и промышленных деталей из этого материала в сверхпроводящем состоянии методами нелинейной акустики. Достоинство этого метода заключается в том, что он дает возможность проводить непрерывную диагностику конструкций, изготовленных из ВТСП керамики, в процессе их эксплуатации.

Проведенные экспериментальные исследования линейных и нелинейных упругих свойств керамики $\mathrm{YBa}_{2} \mathrm{Cu}_{3} \mathrm{O}_{7-x}$ в области сверхпроводящего перехода показывают, что переход в сверхпроводящее состояние оказывает существенное влияние на линейные и нелинейные упругие сдвиговые свойства данного материала.

\section{Благодарности}

Авторы благодарят Круглова В.С. за предоставленные образцы.

\section{Финансирование работы}

Исследования упругих характеристик выполнены за счет гранта Российского научного фонда (проект № 14-22-00042).

\section{Конфликт интересов}

Авторы заявляют, что них нет конфликта интересов.

\section{Список литературы}

[1] Wu M.K., Ashburn J.R., Torng C.J., Hor P.H., Meng R.L., Gao L., Huang Z.J., Wang Y.Q., Chu C.W. // Phys. Rev. Lett. 1987. Vol. 58. N 9. P. $908-910$.

[2] Nikiforov V.N., Bulychev N.A., Rzhevskii V.V. // Bull. Lebedev Phys. Inst. 2016. Vol. 43. N 2. P. 74-79.

[3] Walter H., Bock J., Frohne Ch., Schippl K., May H., Canders W.R., Kummeth P., Nick W., Neumueller H.-W. // J. Phys.: Conf. Series. 2006. Vol. 43. P. 995-998.

[4] Galliano P.G., Soga N., Hirao K. // J. Mater. Sci. 1992. Vol. 27. P. 2621-2626.

[5] Воронов Б.Б., Коробов А.И., Мощалков В.В. // Сверхпроводимость: физика, химия, техника. 1990. Т. 3. Вып. 12. C. 2733-2744.

[6] Воронов Б.Б., Коробов А.И. // Физика низких температур. 1991. Т. 17. Вып. 11-12. С. $1573-1576$.

[7] Гаджимагомедов С.Х., Палчаев Д.К., Рабаданов М.Х., Мурлиева Ж.Х., Шабанов Н.С., Палчаев Н.А., Мурлиев Э.К., Эмиров Р.М. // Письма в ЖТФ. 2016. Т. 42. Вып. 1. C. 9-16. [Gadjimagomedov S., Palchaev D., Rabadanov M., Murlieva Zh., Shabanov N., Palchaev N., Murliev E.K., Emirov R. // Tech. Phys. Lett. 2016. Vol. 42. P. 4-7. DOI: $10.1134 / \mathrm{S} 1063785016010065]$

[8] Белодедов М.В., Черных С.В. // ЖТФ. 2003. Т. 48. Вып. 2. C. 75-80. [Belodedov M.V., Chernykh S.V. // Tech. Phys. 2003. Vol. 48. N 2. P. 209-214.]
[9] Головашкин А.И., Данилов В.А., Иваненко О.М., Мицен К.В., Перепечко И.И. // Письма в ЖЭТФ. 1987. Т. 46. Вып. 7. С. 273-275.

[10] Swenson C.A., McCallum R.W., No K. // Phys. Rev. B. 1989. Vol. 40. N 13. P. 8861-8871.

[11] Руденко О.В. // УФН. 2006. Т. 176. Вып. 1. С. 77-95. [Rudenko O.V. // Phys. Usp. 2006. Vol. 49. N 1. P. 69-87.]

[12] Hull J.R., Murakami M. Proceedings of the IEEE. 2004. Vol. 92. N 10. P. 1705-1718.

[13] Зарембо Л.К., Красильников В.А. Введение в нелинейную акустику. М.: Наука, 1966. 309 с.

[14] Гедройи А.А., Зарембо Л.К., Красильников В.А. // ДАН CCCР. 1963. Т. 150. С. 515-518.

[15] Труэлл Р., Эльбаум Ч., Чик Б. Ультразвуковые методы в физике твердого тела. М.: Мир, 1972. 307 c. [Truell R., Elbaum C., Chick B. Ultrasonic Methods in Solid State Physics. NY:: Academic Press, 1969. 478 p.]

[16] Сиротин Ю.И., Шаскольская М.П. Основы кристаллоакустики. М.: Наука, 1975. 680 с.

[17] Мэзон У. Физическая акустика. Т. 1. Методы и приборы ультразвуковых исследований. М.: Мир, 1966. 588 с. 